(C) 2011 IEEE. Personal use of this material is permitted. Permission from IEEE must be obtained for all other uses, in any current or future media, including reprinting/republishing this material for advertising or promotional purposes, creating new collective works, for resale or redistribution to servers or lists, or reuse of any copyrighted component of this work in other works. 


\title{
Joint Source and Relay Optimization for Two-Way MIMO Multi-Relay Networks
}

\author{
Yue Rong, Senior Member, IEEE
}

\begin{abstract}
In this letter, we study two-way nonregenerative multiple-input multiple-output (MIMO) relay communications with multiple relay nodes. An iterative algorithm is developed to jointly optimize the source, relay, and receive matrices such that the two-way sum mean-squared error (MSE) of the signal waveform estimation is minimized. Numerical examples demonstrate a better performance of the proposed algorithm compared with existing algorithms for two-way MIMO multi-relay networks.
\end{abstract}

Index Terms-MIMO relay, MMSE, Multi-relay system.

\section{INTRODUCTION}

In a two-way relay communication system, two source nodes exchange their information through assisting relay node(s). By using the idea of analog network coding, such information exchange can be accomplished in two time slots even with half-duplex relays [1]. This leads to a high spectral efficiency.

Distributed space-time coding has been designed in [2] for two-way relay communication with multiple single-antenna relay nodes. For a two-way (and in general $N$-way) relay system with a multi-antenna relay node and single-antenna source nodes, the optimal relay matrix has been proposed in [3]-[5] for various objective functions. When all nodes in the network have multiple antennas, we call such system a twoway multiple-input multiple-output (MIMO) relay system. For two-way MIMO relay system with a single relay node, the optimal relay and source matrices have been developed in [6] to maximize the two-way sum mutual information. A minimal mean-squared error (MMSE) based two-way MIMO relay system was proposed in [7]. Two-way relay communication in a multiuser scenario was studied in [8]. Recently, a gradient algorithm has been developed in [9] to optimize the source and relay matrices of two-way MIMO relay systems with multiple relay nodes.

In this letter, we consider two-way nonregenerative MIMO relay communication systems with multiple relay nodes, which include systems in [3]-[7] as special ceases. We aim at minimizing the two-way sum MSE of the signal waveform estimation. Compared with existing works such as [9], the main contribution of this letter lies in that an iterative algorithm with guaranteed convergence is developed to jointly optimize the source, relay, and receive matrices through solving convex subproblems. Simulation results demonstrate that the proposed

Manuscript received July 21, 2011. The associate editor coordinating the review of this letter and approving it for publication was Giovanni Giambene.

Yue Rong is with the Department of Electrical and Computer Engineering, Curtin University, Bentley, WA 6102, Australia (e-mail: y.rong@curtin.edu.au). This research was supported under the Australian Research Council's Discovery Projects funding scheme (project numbers DP110100736, DP110102076) algorithm yields better MSE and bit-error-rate (BER) performance compared with that of [9]. We would like to mention that since one-way relay system can be viewed as a special case of two-way relay system, the proposed iterative algorithm can be applied to optimize the source, relay, and receive matrices in a one-way MIMO relay system with multiple relay nodes [9], [10].

\section{SySTEM MODEL}

We consider a two-way MIMO relay communication system where nodes 1 and 2 exchange information with the aid of $K$ relay nodes. We assume that both nodes 1 and 2 are equipped with $N$ antennas, and each relay node has $L$ antennas. The generalization to a system with different number of antennas at each node is straightforward. The information exchange between nodes 1 and 2 is completed in two time slots. In the first time slot, nodes 1 and 2 concurrently transmit, and the signal vector from node $i$ is $\mathbf{x}_{i}=\mathbf{B}_{i} \mathbf{s}_{i}, i=1,2$, where $\mathbf{s}_{i}$ is the $M_{i} \times 1\left(M_{i} \leq N\right)$ modulated source signal vector, and $\mathbf{B}_{i}$ is the $N \times M_{i}$ source precoding matrix at node $i$. We assume that the source signal vector satisfies $\mathrm{E}\left[\mathbf{s}_{i} \mathbf{s}_{i}^{H}\right]=\mathbf{I}_{M_{i}}$, $i=1,2$, where $\mathbf{I}_{n}$ stands for an $n \times n$ identity matrix, $(\cdot)^{H}$ is the matrix (vector) Hermitian transpose, and $\mathrm{E}[\cdot]$ denotes statistical expectation. The $L \times 1$ received signal vector $\mathbf{y}_{r, k}$ at the $k$ th relay node can be written as

$$
\mathbf{y}_{r, k}=\mathbf{H}_{r 1, k} \mathbf{x}_{1}+\mathbf{H}_{r 2, k} \mathbf{x}_{2}+\mathbf{v}_{r, k}, \quad k=1, \cdots, K
$$

where $\mathbf{H}_{r i, k}$ is the $L \times N$ MIMO channel from node $i$ to the $k$ th relay node and $\mathbf{v}_{r, k}$ is the additive Gaussian noise vector at the $k$ th relay node.

In the second time slot, the $k$ th relay node linearly amplifies $\mathbf{y}_{r, k}$ and broadcasts the signal vector

$$
\mathbf{x}_{r, k}=\mathbf{F}_{k} \mathbf{y}_{r, k}, \quad k=1, \cdots, K
$$

to nodes 1 and 2, where $\mathbf{F}_{k}$ is the $L \times L$ amplifying matrix at the $k$ th relay node. The received signal vector $\mathbf{y}_{i}$ at node $i$ can be written as

$$
\mathbf{y}_{i}=\sum_{k=1}^{K} \mathbf{H}_{i r, k} \mathbf{x}_{r, k}+\mathbf{v}_{i}, \quad i=1,2
$$

where $\mathbf{H}_{i r, k}$ is the $N \times L$ MIMO channel from the $k$ th relay node to node $i$ and $\mathbf{v}_{i}$ is the additive Gaussian noise vector at node $i$.

Substituting (1) and (2) into (3) and after the selfinterference removal we obtain that

$$
\mathbf{y}_{1}=\tilde{\mathbf{H}}_{2} \mathbf{s}_{2}+\tilde{\mathbf{v}}_{2}, \quad \mathbf{y}_{2}=\tilde{\mathbf{H}}_{1} \mathbf{s}_{1}+\tilde{\mathbf{v}}_{1}
$$

where $\tilde{\mathbf{H}}_{i} \triangleq \sum_{k=1}^{K}\left(\mathbf{H}_{\bar{i} r, k} \mathbf{F}_{k} \mathbf{H}_{r i, k}\right) \mathbf{B}_{i}, \quad i=1,2$, is the equivalent MIMO channel between the $i$ th transmit node and 
the $\bar{i}$ th receive node, and $\tilde{\mathbf{v}}_{\bar{i}} \triangleq \sum_{k=1}^{K} \mathbf{H}_{i r, k} \mathbf{F}_{k} \mathbf{v}_{r, k}+\mathbf{v}_{i}$, $i=1,2$, is the equivalent noise vector at node $i$. Here, $\bar{i}=2$ for $i=1$, and $\bar{i}=1$ for $i=2$. We assume that all noises are independent and identically distributed (i.i.d.) Gaussian noise with zero mean and unit variance.

Using linear receivers at both nodes 1 and 2, the estimated signal waveforms are given by $\hat{\mathbf{s}}_{1}=\mathbf{W}_{1}^{H} \mathbf{y}_{2}$ and $\hat{\mathbf{s}}_{2}=\mathbf{W}_{2}^{H} \mathbf{y}_{1}$, where $\mathbf{W}_{i}$ is an $N \times M_{i}$ weight matrix for retrieving $\mathbf{s}_{i}$. From (4), the two-way sum MSE of the signal waveform estimation can be written as

$$
\begin{aligned}
\mathrm{MSE}= & \sum_{i=1}^{2} \operatorname{tr}\left(\mathrm{E}\left[\left(\hat{\mathbf{s}}_{i}-\mathbf{s}_{i}\right)\left(\hat{\mathbf{s}}_{i}-\mathbf{s}_{i}\right)^{H}\right]\right) \\
= & \sum_{i=1}^{2} \operatorname{tr}\left(\left(\mathbf{W}_{i}^{H} \tilde{\mathbf{H}}_{i}-\mathbf{I}_{M_{i}}\right)\left(\mathbf{W}_{i}^{H} \tilde{\mathbf{H}}_{i}-\mathbf{I}_{M_{i}}\right)^{H}\right. \\
& \left.+\mathbf{W}_{i}^{H} \mathbf{C}_{\tilde{v}_{i}} \mathbf{W}_{i}\right)
\end{aligned}
$$

where $\operatorname{tr}(\cdot)$ denotes matrix trace and $\mathbf{C}_{\tilde{v}_{i}}$ is the equivalent noise covariance matrix given by $\mathbf{C}_{\tilde{v}_{i}}=\mathrm{E}\left[\tilde{\mathbf{v}}_{i} \tilde{\mathbf{v}}_{i}^{H}\right]=$ $\sum_{k=1}^{K} \mathbf{H}_{\bar{i} r, k} \mathbf{F}_{k} \mathbf{F}_{k}^{H} \mathbf{H}_{\bar{i} r, k}^{H}+\mathbf{I}_{N}, i=1,2$. From (1) and (2), the transmission power consumed by each relay node is given by $\operatorname{tr}\left(\mathbf{F}_{k}\left(\sum_{i=1}^{2} \mathbf{H}_{r i, k} \mathbf{B}_{i} \mathbf{B}_{i}^{H} \mathbf{H}_{r i, k}^{H}+\mathbf{I}_{L}\right) \mathbf{F}_{k}^{H}\right), k=1, \cdots, K$.

\section{Proposed Source, Relay, and Receive Matrices DESIGN}

The joint source, relay, and receive matrices optimization problem can be written as

$$
\begin{aligned}
\min _{\left\{\mathbf{W}_{i}\right\},\left\{\mathbf{B}_{i}\right\},\left\{\mathbf{F}_{k}\right\}} & \sum_{i=1}^{2} \operatorname{tr}\left(\left(\mathbf{W}_{i}^{H} \tilde{\mathbf{H}}_{i}-\mathbf{I}_{M_{i}}\right)\left(\mathbf{W}_{i}^{H} \tilde{\mathbf{H}}_{i}-\mathbf{I}_{M_{i}}\right)^{H}\right. \\
& \left.+\mathbf{W}_{i}^{H} \mathbf{C}_{\tilde{v}_{i}} \mathbf{W}_{i}\right) \\
\text { s.t. } & \operatorname{tr}\left(\mathbf{F}_{k}\left(\sum_{i=1}^{2} \mathbf{H}_{r i, k} \mathbf{B}_{i} \mathbf{B}_{i}^{H} \mathbf{H}_{r i, k}^{H}+\mathbf{I}_{L}\right) \mathbf{F}_{k}^{H}\right) \\
& \leq P_{k}, \quad k=1, \cdots, K \\
& \operatorname{tr}\left(\mathbf{B}_{i} \mathbf{B}_{i}^{H}\right) \leq Q_{i}, \quad i=1,2
\end{aligned}
$$

where $\left\{\mathbf{F}_{k}\right\} \triangleq\left\{\mathbf{F}_{k}, k=1, \cdots, K\right\},\left\{\mathbf{W}_{i}\right\} \triangleq\left\{\mathbf{W}_{1}, \mathbf{W}_{2}\right\}$, $\left\{\mathbf{B}_{i}\right\} \triangleq\left\{\mathbf{B}_{1}, \mathbf{B}_{2}\right\},(7)$ and (8) are the transmission power constraints at the relay nodes and the source nodes, respectively, and $P_{k}, k=1, \cdots, K, Q_{i}, i=1,2$, are the corresponding power available.

The problem (6)-(8) is a nonconvex problem with matrix variables. The global optimum is difficult to obtain with a reasonable computational complexity (non-exhaustive searching). In the following, we develop an iterative algorithm to solve the problem (6)-(8). This algorithm starts at a random $\left\{\mathbf{F}_{k}\right\}$ and $\left\{\mathbf{B}_{i}\right\}$ satisfying (7) and (8). In each iteration, the source, relay, and receive matrices are updated alternatingly through solving convex subproblems. First, with given $\left\{\mathbf{F}_{k}\right\}$ and $\left\{\mathbf{B}_{i}\right\}$, the optimal $\left\{\mathbf{W}_{i}\right\}$ are obtained by solving the unconstrained convex problem (6), since $\left\{\mathbf{W}_{i}\right\}$ do not appear in constraints (7) and (8). The solution is given by

$$
\mathbf{W}_{i}=\left(\tilde{\mathbf{H}}_{i} \tilde{\mathbf{H}}_{i}^{H}+\mathbf{C}_{\tilde{v}_{i}}\right)^{-1} \tilde{\mathbf{H}}_{i}, \quad i=1,2
$$

where $(\cdot)^{-1}$ denotes matrix inversion.
Second, with fixed $\left\{\mathbf{W}_{i}\right\},\left\{\mathbf{B}_{i}\right\}$, and $\mathbf{F}_{j}, j=1, \cdots, K, j \neq$ $k$, the optimal $\mathbf{F}_{k}$ can be obtained by solving the problem of

$$
\begin{aligned}
\min _{\mathbf{F}_{k}} & \sum_{i=1}^{2} \operatorname{tr}\left(\left(\mathbf{H}_{i, k} \mathbf{F}_{k} \mathbf{G}_{i, k}-\mathbf{A}_{i, k}\right)\left(\mathbf{H}_{i, k} \mathbf{F}_{k} \mathbf{G}_{i, k}-\mathbf{A}_{i, k}\right)^{H}\right. \\
& \left.+\mathbf{H}_{i, k} \mathbf{F}_{k} \mathbf{F}_{k}^{H} \mathbf{H}_{i, k}^{H}\right) \\
\text { s.t. } & \operatorname{tr}\left(\mathbf{F}_{k}\left(\sum_{i=1}^{2} \mathbf{G}_{i, k} \mathbf{G}_{i, k}^{H}+\mathbf{I}_{L}\right) \mathbf{F}_{k}^{H}\right) \leq P_{k}
\end{aligned}
$$

where $\mathbf{H}_{i, k} \triangleq \mathbf{W}_{i}^{H} \mathbf{H}_{\bar{i} r, k}, \mathbf{G}_{i, k} \triangleq \mathbf{H}_{r i, k} \mathbf{B}_{i}$, and $\mathbf{A}_{i, k} \triangleq \mathbf{I}_{M_{i}}-$ $\mathbf{W}_{i}^{H} \sum_{j=1, j \neq k}^{K}\left(\mathbf{H}_{\bar{i} r, j} \mathbf{F}_{j} \mathbf{H}_{r i, j}\right) \mathbf{B}_{i}$. The structure of $\mathbf{F}_{k}$ can be derived by using the Lagrange multiplier method [11] to solve the problem (9)-(10). After rearranging of terms, the optimal $\mathbf{f}_{k} \triangleq \operatorname{vec}\left(\mathbf{F}_{k}\right)$ can be written as

$$
\begin{aligned}
& \mathbf{f}_{k}=\left[\sum_{i=1}^{2}\left(\mathbf{G}_{i, k} \mathbf{G}_{i, k}^{H}+\mathbf{I}_{L}\right)^{T} \otimes\left(\mathbf{H}_{i, k}^{H} \mathbf{H}_{i, k}\right)\right. \\
& \left.+\lambda_{k}\left(\sum_{i=1}^{2}\left(\mathbf{G}_{i, k} \mathbf{G}_{i, k}^{H}\right)+\mathbf{I}_{L}\right)^{T} \otimes \mathbf{I}_{L}\right]^{-1} \operatorname{vec}\left(\sum_{i=1}^{2} \mathbf{H}_{i, k}^{H} \mathbf{A}_{i, k} \mathbf{G}_{i, k}^{H}\right)(11)
\end{aligned}
$$

where $\operatorname{vec}(\cdot)$ stands for a vector obtained by stacking all column vectors of a matrix on top of each other, $(\cdot)^{T}$ denotes the matrix (vector) transpose, and $\lambda_{k} \geq 0$ is the unknown Lagrangian multiplier which can be found as follows. Assuming $\lambda_{k}=0$, we have

$$
\begin{aligned}
\mathbf{f}_{k}=\left[\sum_{i=1}^{2}\left(\mathbf{G}_{i, k} \mathbf{G}_{i, k}^{H}+\mathbf{I}_{L}\right)^{T} \otimes\left(\mathbf{H}_{i, k}^{H} \mathbf{H}_{i, k}\right)\right]^{-1} \\
\times \operatorname{vec}\left(\sum_{i=1}^{2} \mathbf{H}_{i, k}^{H} \mathbf{A}_{i, k} \mathbf{G}_{i, k}^{H}\right) .
\end{aligned}
$$

If $\mathbf{f}_{k}$ in (12) satisfies the constraint (10), then (12) is the solution to the problem (9)-(10). Otherwise $\lambda_{k}>0$ can be obtained by substituting (11) into the equation of $\operatorname{tr}\left(\mathbf{F}_{k}\left(\sum_{i=1}^{2} \mathbf{G}_{i, k} \mathbf{G}_{i, k}^{H}+\mathbf{I}_{L}\right) \mathbf{F}_{k}^{H}\right)=P_{k}$. Since the lefthand side of the obtained nonlinear equation is monotonically decreasing with respect to $\lambda_{k}$, it can be efficiently solved using the bisection method [11].

Finally, using given $\left\{\mathbf{W}_{i}\right\}$ and $\left\{\mathbf{F}_{k}\right\}$, we update $\left\{\mathbf{B}_{i}\right\}$ by solving the following problem

$$
\begin{aligned}
\min _{\left\{\mathbf{B}_{i}\right\}} & \sum_{i=1}^{2} \operatorname{tr}\left(\left(\tilde{\mathbf{W}}_{i} \mathbf{B}_{i}-\mathbf{I}_{M_{i}}\right)\left(\tilde{\mathbf{W}}_{i} \mathbf{B}_{i}-\mathbf{I}_{M_{i}}\right)^{H}\right) \\
\text { s.t. } & \sum_{i=1}^{2} \operatorname{tr}\left(\mathbf{F}_{k} \mathbf{H}_{r i, k} \mathbf{B}_{i} \mathbf{B}_{i}^{H} \mathbf{H}_{r i, k}^{H} \mathbf{F}_{k}^{H}\right) \leq \tilde{P}_{k}, k=1, \cdots, K \\
& \operatorname{tr}\left(\mathbf{B}_{i} \mathbf{B}_{i}^{H}\right) \leq Q_{i}, \quad i=1,2
\end{aligned}
$$

where $\tilde{\mathbf{W}}_{i} \triangleq \mathbf{W}_{i}^{H} \sum_{k=1}^{K}\left(\mathbf{H}_{\bar{i} r, k} \mathbf{F}_{k} \mathbf{H}_{r i, k}\right)$ and $\tilde{P}_{k} \triangleq P_{k}$ $\operatorname{tr}\left(\mathbf{F}_{k} \mathbf{F}_{k}^{H}\right), k=1, \cdots, K$. The problem (13)-(15) is a quadratically constrained quadratic programming (QCQP) problem [11], which is a convex optimization problem and can be efficiently solved by the interior-point method [11]. Since all subproblems (6), (9)-(10), and (13)-(15) are convex, the solution to each subproblem is optimal. Thus, the value of the objective function (6) decreases after each iteration. Moreover, 
the objective function is lower bounded by at least zero. Therefore, the iterative algorithm converges. It can be easily shown that at the convergence point $\mathbf{X}_{0}, \operatorname{tr}\left(\nabla J\left(\mathbf{X}_{0}\right)^{T}\left(\mathbf{X}-\mathbf{X}_{0}\right)\right) \geq 0$, where $\mathbf{X} \triangleq\left[\left\{\mathbf{W}_{i}\right\},\left\{\mathbf{B}_{i}\right\},\left\{\mathbf{F}_{k}\right\}\right]$ and $\nabla J\left(\mathbf{X}_{0}\right)$ is the gradient of (6) at $\mathbf{X}_{0}$. Moreover, it can be seen that $\mathbf{X}_{0}$ must be on the edge of the feasible set specified by inequalities in (7) and (8). This indicates that $\mathbf{X}_{0}$ can not be a saddle point and is indeed the local-optimal solution. Therefore, the proposed iterative algorithm monotonically converges to (at least) a locally optimal solution.

\section{NumericAl EXAMPlES}

We simulate a two-way MIMO relay system with $N=$ $K=L=3$ and $M_{1}=M_{2}=M$. All channel matrices have i.i.d. complex Gaussian entries with zero-mean and variances $1 / N$ for $\mathbf{H}_{r i, k}, i=1,2, k=1, \cdots, K$, and $1 / L$ for $\mathbf{H}_{i r, k}, i=1,2, k=1, \cdots, K$, respectively. The QPSK constellations are used to modulate the source symbols. All noises are i.i.d. Gaussian with zero mean and unit variance. We set $Q_{1}=Q_{2}=20 \mathrm{~dB}$ and $P_{k}=P, k=1, \cdots, K$. The CVX convex optimization software package [12] is applied to solve the QCQP problem (13)-(15). All simulation results are averaged over 1000 independent channel realizations.

We compare the performance of the proposed joint source, relay, and receive matrices design algorithm and the gradient algorithm developed in [9] using the MSE objective. Fig. 1 shows the MSE performance of both algorithms versus $P$ for $M=2$. It can be clearly seen that the performance of the proposed algorithm is similar to that of [9] after 5 iterations. And after 10 iterations, the proposed algorithm outperforms [9] especially at large $P$. The reason is that for the gradient algorithm in [9], the source and relay matrices are essentially updated in two steps. First, they are modified by moving one step towards the gradient descent direction of the objective function, without considering any power constraint at each node. Then the power constraint is imposed by scaling the source and relay matrices. Such two-step scheme is suboptimal in terms of power loading at all data streams. While in the proposed algorithm, the source and relay matrices are updated directly considering the power constraints in each step, which is optimal in power allocation. We observed in simulations that for most channel realizations, the decreasing of the objective function (6) is negligible after 10 iterations. Thus, for the proposed algorithm, only a small number of iterations are required to achieve a good performance. On the other hand, the convergence of the gradient algorithm strongly depends on the choice of the step size at each iteration.

The BER performance of both algorithms for $M=2$ and $M=3$ is shown in Fig. 2. It can be clearly seen that the proposed algorithm has a better performance than that of [9]. From Fig. 2 we can also see that due to the increase of effective spatial diversity, both algorithms yield smaller BER when $M$ reduces from three to two.

\section{CONCLUSION}

We have studied two-way nonregenerative MIMO relay communication systems with multiple relay nodes. A joint source, relay, and receive matrices optimization algorithm has

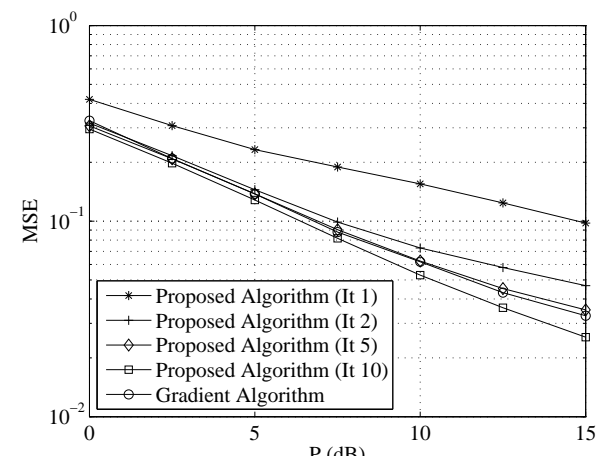

Fig. 1. MSE versus $P . K=3, L \stackrel{\mathrm{P}(\mathrm{dB})}{=}, N=3, M=2, Q=20 \mathrm{~dB}$.

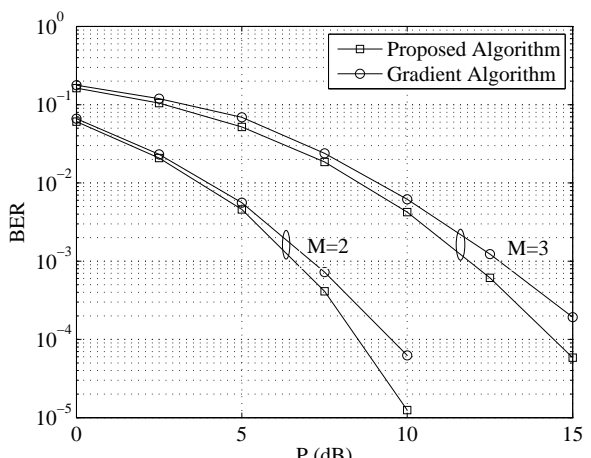

Fig. 2. BER versus $P . K=3, L \stackrel{\mathrm{P}(\mathrm{dB})}{=}, N=3, Q=20 \mathrm{~dB}$.

been developed to minimize the two-way sum MSE of the signal waveform estimation.

\section{REFERENCES}

[1] S. Katti, S. Gollakota, and D. Katabi, "Embracing wireless interference: Analog network coding," in Proc. ACM SIGCOMM, Kyoto, Japan, Aug. 27-31, 2007.

[2] T. Cui, F. Gao, T. Ho, and A. Nallanathan, "Distributed space-time coding for two-way wireless relay networks," in Proc. IEEE ICC, Beijing, China, May 19-23, 2008, pp. 3888-3892.

[3] R. Zhang, Y.-C. Liang, C. C. Chai, and S. Cui, "Optimal beamforming for two-way multi-antenna relay channel with analogue network coding," IEEE J. Sel. Areas Commun., vol. 27, pp. 699-712, Jun. 2009.

[4] F. Gao, T. Cui, B. Jiang, and X. Gao, "On communication protocol and beamforming design for amplify-and-forward N-way relay networks," in Proc. IEEE CAMSAP, Aruba, Dutch Antilles, Dec. 2009. pp. 109-112.

[5] F. Roemer and M. Haardt, "Sum-rate maximization in two-way relaying systems with MIMO amplify and forward relays via generalized eigenvectors," in Proc. EUSIPCO, Aalborg, Denmark, Aug. 2010, pp. 377381.

[6] S. Xu and Y. Hua, "Source-relay optimization for a two-way MIMO relay system," in Proc. IEEE ICASSP, Dallas, TX, USA, Mar. 2010.

[7] R. Wang and M. Tao, "Joint source and relay precoding designs for MIMO two-way relay systems," in Proc. IEEE ICC, Kyoto, Japan, Jun. 5-9, 2011.

[8] J. Joung and A. H. Sayed, "Multiuser two-way amplify-and-forward relay processing and power control methods for beamforming systems," IEEE Trans. Signal Process., vol. 58, pp. 1833-1846, Mar. 2010.

[9] K.-J. Lee, H. Sung, E. Park, and I. Lee, "Joint optimization for one and two-way MIMO AF multiple-relay systems," IEEE Trans. Wireless Commun., vol. 9, pp. 3671-3681, Dec. 2010.

[10] A. S. Behbahani, R. Merched, and A. M. Eltawil, "Optimizations of a MIMO relay network,” IEEE Trans. Signal Process., vol. 56, pp. 50625073, Oct. 2008.

[11] S. Boyd and L. Vandenberghe, Convex Optimization. Cambridge, U. K.: Cambridge University Press, 2004.

[12] M. Grant and S. Boyd, "Cvx: Matlab software for disciplined convex programming (webpage and software)," [Online]. Available: http://cvxr.com/cvx, April, 2010. 